\title{
Examining Coupled-Channel Effects in Radiative Charmonium Transitions
}

\author{
Feng-Kun Guo ${ }^{1, *}$ and Ulf-G. Meißner ${ }^{1,2, \dagger}$ \\ ${ }^{1}$ Helmholtz-Institut für Strahlen- und Kernphysik and Bethe Center for Theoretical Physics, Universität Bonn, \\ D-53115 Bonn, Germany \\ ${ }^{2}$ Institute for Advanced Simulation, Institut für Kernphysik and Jülich Center for Hadron Physics,
}

Forschungszentrum Jülich, D-52425 Jülich, Germany

(Received 8 November 2011; published 13 March 2012)

\begin{abstract}
Coupled-channel effects due to coupling of charmonia to the charmed and anticharmed mesons are of current interest in heavy quarkonium physics. However, the effects have not been unambiguously established. In this Letter, a clean method is proposed in order to examine the coupled-channel effects in charmonium transitions. We show that the hindered $M 1$ radiative transitions from the $2 P$ to $1 P$ charmonia are suitable for this purpose. We suggest to measure one or more of the ratios $\Gamma\left(h_{c}^{\prime} \rightarrow \chi_{c J} \gamma\right) / \Gamma\left(\chi_{c J}^{\prime} \rightarrow \chi_{c J} \pi^{0}\right)$ and $\Gamma\left(\chi_{c J}^{\prime} \rightarrow h_{c} \gamma\right) / \Gamma\left(\chi_{c J}^{\prime} \rightarrow \chi_{c J} \pi^{0}\right)$, for which highly nontrivial and parameter-free predictions are given. The picture can also be tested using both unquenched and quenched lattice calculations.
\end{abstract}

DOI: 10.1103/PhysRevLett.108.112002

PACS numbers: 13.25.Gv, $14.40 . \mathrm{Pq}$

Thanks to various experiments world-wide, our knowledge of the physics of heavy quarkonium has been greatly enriched in the last decade. New charmonium(-like) states, including the $h_{c}$ and $\chi_{c 2}^{\prime}$ as well as the so-called $X Y Z$ states, were observed. Most of the $X Y Z$ states are above the open-charm thresholds, and do not fit the expectations from the quark model. Hence, it is of current interest and high importance to investigate the coupled-channel effects, originating from the coupling of $c \bar{c}$ to charmed-meson-anticharmed-meson channels, in charmonium physics. So far, these effects have not been established unambiguously, though evidences exist, see, e.g., Refs. [1-3] for transitions between charmonia and Refs. [4,5] for spectroscopy. This Letter is devoted to a clean way of examining the coupledchannel effects. For this purpose, we propose to measure one or more of the ratios $\Gamma\left(h_{c}^{\prime} \rightarrow \chi_{c J} \gamma\right) / \Gamma\left(\chi_{c J}^{\prime} \rightarrow \chi_{c J} \pi^{0}\right)$ and $\Gamma\left(\chi_{c J}^{\prime} \rightarrow h_{c} \gamma\right) / \Gamma\left(\chi_{c J}^{\prime} \rightarrow \chi_{c J} \pi^{0}\right)$, for which highly nontrivial predictions will be made.

At the hadronic level, one may consider the coupling of heavy quarkonium states to open-flavor mesons and antimesons using effective Lagrangians, and take into account the coupled-channel effects by calculating intermediate heavy meson loops. Because the difference between the mass of a heavy quarkonium and heavy meson-antimeson thresholds is small, the intermediate heavy mesons are nonrelativistic with velocity $v \ll 1$. Based on this observation, a nonrelativistic effective field theory (NREFT) was proposed $[1,6,7]$. It was found that the transitions between two $P$-wave charmonia with the emission of one pion are completely dominated by the coupled-channel effects with an enhancement of $\sim 1 / v^{3}$ in the decay amplitude in contrast to the multipole contribution [1]. Here we find that the hindered $M 1$ transitions from a $2 P$ to a $1 P$ charmonium is also dominated by the coupled-channel effects. Because these two types of transitions cannot be directly connected to each other, only when both of them are dominated by the coupled-channel effects, nontrivial predictions can be made in the framework of NREFT. Consequently, the measurements of hindered $M 1$ transitions from $P$-wave charmonia provides a good opportunity for examining the coupledchannel effects in charmonium transitions.

There are several nice features of the hindered $M 1$ transitions of $P$-wave charmonia for investigating the coupled-channel effects of open-charm mesons.

(i) First of all, these transitions are expected to be dominated by the coupled-channel effects. On one hand, in quark models, the decay amplitude for an $M 1$ transition between two heavy quarkonia is proportional to the overlap of the wave functions of the initial and final quarkonia (see, e.g., [8]),

$$
\Gamma_{M 1} \propto\left|\left\langle\psi_{f} \mid \psi_{i}\right\rangle\right|^{2} E_{\gamma}^{3},
$$

with $\psi_{i(f)}$ being the wave function of the initial (final) heavy quarkonium, and $E_{\gamma}$ the photon energy in the rest frame of the initial particle. For transitions between a $2 P$ and a $1 P$ state, if the charmonia are purely $c \bar{c}$ states, the overlap is nonzero only because of small relativistic corrections. The statement can be made model-independently using the potential nonrelativistic QCD [9]—the leading contribution vanishes [10]. Hence, the transition amplitude would start from $E_{\gamma} v_{c} / m_{c}$, where $v_{c}$ and $m_{c}$ are the charm quark velocity and mass, respectively, and the factor $1 / m_{c}$ accounts for the spin-flip. Indeed, the transition rates are very small in quark model calculations-at the largest of the order $1 \mathrm{keV}$ [8]. On the other hand, because the leading coupling of a $P$-wave charmonium to a charmed-meson and anticharmed-meson pair is in an $S$ wave, the decay amplitude through intermediate charmed-meson loops as shown in Fig. 1(a) scales as

$$
\mathcal{A}_{(a)} \sim \frac{v^{5}}{\left(v^{2}\right)^{3}} \frac{E_{\gamma}}{m_{c}}=\frac{E_{\gamma}}{m_{c} v},
$$




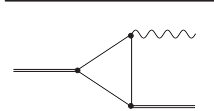

(a) (b)

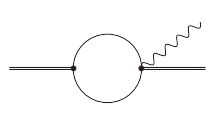

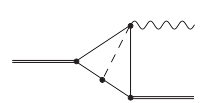

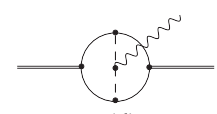

(d)
FIG. 1. Possible triangle (a) and two-point (b) loops for the radiative transitions. (c) and (d) are two typical two-loop diagrams. The double, solid, wavy, and dashed lines represent charmonia, charmed mesons, photons, and pion, respectively.

where $v^{5}$ and $\left(v^{2}\right)^{3}$ account for the nonrelativistic loop measure and three nonrelativistic propagators, respectively, and $E_{\gamma}$ comes from the $P$-wave coupling of the photon to charmed mesons. The factor of $1 / m_{c}$ is again due to the spin-flip [more details will be given below Eq. (8)]. In addition, the amplitude is proportional to the electric charge $e$ and the product of coupling constants of the $1 P$ and $2 P$ states to the charmed mesons. Yet, there is no suppression analogous to $\left|\left\langle\psi_{f} \mid \psi_{i}\right\rangle\right|^{2}$ because the initial and final charmonia, though having different principal quantum numbers, do not couple to each other directly. Instead, they couple through intermediate charmed mesons, and there is no similar suppression for such couplings. Note that $v$ in Eq. (2) will not approach 0 even when the charmonium mass overlaps with the charmedmeson threshold, since it should be understood as the average of two velocities corresponding to the two cuts in the three-point loop. (ii) The triangle hadronic loops involved in the transitions are convergent in the nonrelativistic framework. Therefore, we do not need to introduce a counterterm. On the contrary, similar loops for the $M 1$ transitions of $S$-wave charmonia are divergent so that similar statements cannot be made there. This is another nice example of the important role of such diagrams in hadron physics, see, e.g., the classical work on neutral pion photoproduction off nucleons [11] or the more recent investigation of the large isospin violation in the decay $\eta(1405 / 1475) \rightarrow 3 \pi$ [12]. (iii) Because the leading coupling of charmed and anticharmed mesons to the $P$-wave charmonium is in an $S$-wave, there is no derivative in such vertices. Hence the two-point loop with the four-particle contact term $\chi_{c J} D^{(*)} \bar{D}^{(*)} \gamma$, as shown in Fig. 1(b), is not related to that in Fig. 1(a) by gauge symmetry. It can be treated separately. Being gauge-invariant by itself, the four-particle contact term should contain the electromagnetic field strength $F^{\mu \nu}$ for the photon. Hence, the corresponding vertex is proportional to the external momentum of the photon. The amplitude for the diagram scales as

$$
\mathcal{A}_{(b)} \sim \frac{v^{5}}{\left(v^{2}\right)^{2}} \frac{E_{\gamma}}{m_{c}}=v \frac{E_{\gamma}}{m_{c}} .
$$

One sees that it is two orders higher in the meson velocity counting than the diagram Fig. 1(a), and hence can be neglected at leading one-loop order. (iv) As will be shown later, the two-loop diagrams (c) and (d) are also suppressed compared with (a).
The coupling of the $P$-wave charmonia to the charmed and anticharmed mesons is described by the Lagrangian [13]

$$
\mathcal{L}_{\chi}=i \frac{g_{1}}{2} \operatorname{Tr}\left[\chi^{\dagger i} H_{a} \sigma^{i} \bar{H}_{a}\right]+\text { H.c. },
$$

where $g_{1}$ is the coupling constant of the $1 P$ charmonium states ( $g_{1}^{\prime}$ will be used for the $2 P$ states), $H_{a}=\vec{V}_{a} \cdot \vec{\sigma}+$ $P_{a}$ and $\bar{H}_{a}=-\overrightarrow{\bar{V}}_{a} \cdot \vec{\sigma}+\bar{P}_{a}$ are fields annihilating charmed and anticharmed mesons, respectively, with $\vec{\sigma}$ the Pauli matrices and $a$ the light flavor index. The twocomponent notation introduced in Ref. [14] is used here, which is convenient for processes with negligible recoil effect (less than $1 \%$ for the processes considered in this Letter). The $P$-wave charmonia are collected in the spinmultiplet

$$
\chi^{i}=\sigma^{j}\left(-\chi_{c 2}^{i j}-\frac{1}{\sqrt{2}} \epsilon^{i j k} \chi_{c 1}^{k}+\frac{1}{\sqrt{3}} \delta^{i j} \chi_{c 0}\right)+h_{c}^{i} .
$$

The magnetic coupling of the photon to heavy mesons is described by the Lagrangian $[14,15]$

$$
\mathcal{L}_{\gamma}=\frac{e \beta}{2} \operatorname{Tr}\left[H_{a}^{\dagger} H_{b} \vec{\sigma} \cdot \vec{B} Q_{a b}\right]+\frac{e Q^{\prime}}{2 m_{Q}} \operatorname{Tr}\left[H_{a}^{\dagger} \vec{\sigma} \cdot \vec{B} H_{a}\right]
$$

where $B^{k}=\epsilon^{i j k} \partial^{i} A^{j}$ is the magnetic field, $Q=$ $\operatorname{diag}\{2 / 3,-1 / 3,-1 / 3\}$ is the light quark charge matrix, and $Q^{\prime}$ is the heavy quark electric charge (in units of $e$ ). The first term describes the nonperturbative physics of the light quarks, while the second term is the magnetic coupling of the heavy mesons and hence is proportional to $1 / m_{Q}$. Although the photon can also couple to the heavy mesons through gauging the kinetic energy term, this vertex does not contribute to the magnetic transitions.

Because the $\chi_{c J}$ states are easier to be detected than the $h_{c}$, and $\chi_{c 2}^{\prime}$ has been observed, we will calculate the decay widths of the hindered $M 1$ transitions $h_{c}^{\prime} \rightarrow \gamma \chi_{c J}$ and $\chi_{c 2}^{\prime} \rightarrow \gamma h_{c}$. Results for the other hindered $M 1$ transitions of the $P$-wave charmonia can be easily obtained using the same method. Denoting the charmed-meson connecting the initial charmonium and the photon as $M 1$, the one connecting two charmonia as $M 2$, and the other as $M 3$, we specify the triangle loops by $[M 1, M 2, M 3]$. Considering both the pseudoscalar and vector charmed mesons, possible loops for these transitions are listed in Table I (see also Ref. [1] for details).

The decay amplitude for each transition can be expressed in terms of the scalar three-point loop function

TABLE I. Possible loops contributing to each transition. The charge-conjugated ones and the flavor labels are not shown for simplicity.

\begin{tabular}{lc}
\hline \hline$h_{c}^{\prime} \rightarrow \gamma \chi_{c 0}$ & {$\left[D \bar{D}^{*}, D^{*}\right],\left[D^{*}, \bar{D}, D\right],\left[D^{*}, \bar{D}^{*}, D^{*}\right]$} \\
$h_{c}^{\prime} \rightarrow \gamma \chi_{c 1}$ & {$\left[D, \bar{D}^{*}, D\right],\left[D^{*}, \bar{D}, D^{*}\right],\left[D^{*}, \bar{D}^{*}, D\right]$} \\
$h_{c}^{\prime} \rightarrow \gamma \chi_{c 2}$ & {$\left[D, \bar{D}^{*}, D^{*}\right],\left[D^{*}, \bar{D}^{*}, D^{*}\right]$} \\
$\chi_{c 2}^{\prime} \rightarrow \gamma h_{c}$ & {$\left[D^{*}, \bar{D}^{*}, D\right],\left[D^{*}, \bar{D}^{*}, D^{*}\right]$} \\
\hline \hline
\end{tabular}




$$
I(q) \equiv i \int \frac{d^{4} l}{(2 \pi)^{4}} \frac{1}{\left(l^{2}-m_{1}^{2}+i \epsilon\right)\left[(P-l)^{2}-m_{2}^{2}+i \epsilon\right]\left[(l-q)^{2}-m_{3}^{2}+i \epsilon\right]},
$$

where $P$ and $q$ are the momenta of the initial particle and the photon, respectively, $m_{i}(i=1,2,3)$ are the masses of the particles $M i$ in the loop. The analytic expression can be found in Refs. [1,16]. The amplitude for the transition $\chi_{c 2}^{\prime} \rightarrow \gamma h_{c}$ reads

$$
\begin{aligned}
\mathcal{A}\left(\chi_{c 2}^{\prime} \rightarrow \gamma h_{c}\right)= & \frac{4 i e g_{1} g_{1}^{\prime}}{3} \epsilon^{i j k} \varepsilon^{k l}\left(\chi_{c 2}^{\prime}\right)\left\{q^{i} \varepsilon^{j}(\gamma) \varepsilon^{l}\left(h_{c}\right)\left[-\left(\beta+\frac{4}{m_{c}}\right) I\left(q, D^{*}, D^{*}, D\right)+\left(\beta-\frac{2}{m_{c}}\right) I\left(q, D_{s}^{*}, D_{s}^{*}, D_{s}\right)\right]\right. \\
& \left.+\left[q^{i} \varepsilon^{j}\left(h_{c}\right) \varepsilon^{l}(\gamma)+q^{l} \varepsilon^{i}\left(h_{c}\right) \varepsilon^{j}(\gamma)\right]\left[\left(\beta-\frac{4}{m_{c}}\right) I\left(q, D^{*}, D^{*}, D^{*}\right)-\left(\beta+\frac{2}{m_{c}}\right) I\left(q, D_{s}^{*}, D_{s}^{*}, D_{s}^{*}\right)\right]\right\},
\end{aligned}
$$

where the loop function has been written as $I(q, M 1, M 2, M 3)$. The charge-conjugated channels are taken into account. (They were not considered in Refs. $[1,6,7,17]$. Hence all the loop amplitudes therein should be doubled, and the decay widths from the loops should be multiplied by 4 . All ratios remain unchanged.) The amplitudes for the other transitions can be obtained similarly.

Since the spin direction of the $c$ or $\bar{c}$ quark should be flipped in the $M 1$ transitions, the decay amplitude should vanish in the heavy quark limit. It is nonzero only because of the $\mathcal{O}\left(m_{c}^{-1}\right)$ spin symmetry breaking effect. One easily sees there must be nonvanishing contributions from the second term in the Lagrangian Eq. (6). In fact, the first term, to be called the $\beta$ term in the following, also contributes at the same order though $m_{c}^{-1}$ is not explicit in the amplitude. Let us look at the decay amplitude given in Eq. (8). The $\beta$-term contribution would vanish were spin symmetry a good symmetry; i.e., different loops proportional to $\beta$ cancel each other exactly if the hyperfine splitting between vector and pseudoscalar charmed mesons $M_{D_{(s)}}-M_{D_{(s)}^{*}}$ is tuned to zero. The surviving part is due to the nonvanishing hyperfine splitting which is of order $m_{c}^{-1}$.

Because the expansion parameter in the NREFT $v \simeq 0.4$ is not small, the results should have sizeable uncertainties. This can be seen by analyzing the power counting of the decay amplitudes for certain two-loop diagrams. In Ref. [16], it is argued that vertex corrections due to pionexchange is suppressed, so that the largest two-loop contribution comes from diagrams shown in Figs. 1(c) and 1(d). The diagram (c) contains four nonrelativistic charmed-meson propagators and one relativistic pion propagator. Each momentum is of order $M_{D} v$, so that each propagator scales as $1 / v^{2}$ in the velocity counting. The photon vertex comes from gauging the charmedmeson-pion-axial coupling; hence, it contributes a factor of $g / F_{\pi}$ with $g$ and $F_{\pi}$ being the axial coupling constant and pion decay constant, respectively. The charmedmeson-pion axial coupling is in a $P$ wave. Because this is the only $P$-wave vertex in the diagram, it should scales as the photon momentum, and the vertex is proportional to
$E_{\gamma} g / F_{\pi}$. Therefore, the decay amplitude for the diagram shown in Fig. 1(c) scales as

$$
\mathcal{A}_{(c)} \sim \frac{\left(v^{5}\right)^{2}}{\left(v^{2}\right)^{5}} \frac{g^{2}}{(4 \pi)^{2} F_{\pi}^{2}} \frac{E_{\gamma}}{m_{c}} M_{D}^{2}=\frac{E_{\gamma}}{m_{c}}\left(\frac{g M_{D}}{\Lambda_{\chi}}\right)^{2},
$$

where the factor $1 /(4 \pi)^{2}$ appears because there is one more loop than in the one-loop case, and the chiral symmetry breaking scale is $\Lambda_{\chi}=4 \pi F_{\pi}$. In order to compare with Eq. (2), a factor of $M_{D}^{2}$ is introduced, with $M_{D}$ being the charmed-meson mass, to make the whole scaling have the same dimension as that in Eq. (2). The value of the axial coupling constant $g=0.6$ can be determined from $\Gamma\left(D^{*} \rightarrow D \pi\right)[18,19]$. Numerically, one has $g M_{D} / \Lambda_{\chi} \simeq$ 1. The diagram (d) has the same scaling as (c). This can be seen easily because the one more propagator in (d) is balanced by two more $P$-wave vertices. Therefore, the two-loop diagrams are effectively suppressed compared with Fig. 1(a) by a factor of $v \simeq 0.4$.

In numerical calculations, we use the central values of all measured masses [19]. The value of $\beta$ is not precisely known. Here, we take the value $\beta^{-1}=276 \mathrm{MeV}$ determined with $m_{c}=1.5 \mathrm{GeV}$ in Ref. [14]. In fact, the precise value of $\beta$ is not important. A change of $\beta^{-1}$ from 276 to $376 \mathrm{MeV}$ only causes a change in the decay width of less than $10 \%$. Hence, the decay width for the $\chi_{c 2}^{\prime} \rightarrow \gamma h_{c}$ is

$$
\Gamma\left(\chi_{c 2}^{\prime} \rightarrow \gamma h_{c}\right)=(10.7 \pm 4.3) \frac{\left(g_{1} g_{1}^{\prime}\right)^{2}}{\mathrm{GeV}^{-2}} \mathrm{keV},
$$

where a $40 \%$ uncertainty has been assigned to account for higher order effects. Because the $\chi_{c J}$ and $h_{c}$ are below the open-charm thresholds, the coupling constant $g_{1}$ cannot be measured directly through the decays of the $P$-wave charmonia. Similarly, since the only established $2 P$ charmonium $\chi_{c 2}^{\prime}$ is below the $D^{*} \bar{D}^{*}$ threshold, $g_{1}^{\prime}$ is also not known yet. In order to obtain an order-of-magnitude estimate of the branching fraction for the decay $\chi_{c 2}^{\prime} \rightarrow$ $\gamma h_{c}$, we take a model value $g_{1} \simeq-4 \mathrm{GeV}^{-1 / 2}$ [13] (The value of $g_{1}$ as defined in Eq. (4) is twice of that in [13]). For $g_{1}^{\prime}$, we resort to quark model calculations of the decay widths of $\chi_{c J}^{\prime}$ and $h_{c}^{\prime}$. From the results in the 
nonrelativistic potential model in Ref. [8], we get $g_{1}^{\prime}=$ $0.5 \ldots 1.3 \mathrm{GeV}^{-1 / 2}$. From the results in the Cornell coupled-channel model [4], we get $g_{1}^{\prime}=$ $0.7 \ldots 1.2 \mathrm{GeV}^{-1 / 2}$. Hence, one may take $g_{1}^{\prime} \simeq$ $1 \mathrm{GeV}^{-1 / 2}$ as an estimate. Thus, we get the estimate $\Gamma\left(\chi_{c 2}^{\prime} \rightarrow \gamma h_{c}\right)=\mathcal{O}(170 \mathrm{keV})$, which is much larger than the quark model prediction $1.3 \mathrm{keV}$ [8]. This is consistent with the analysis made above that the transitions are dominated by the coupled-channel effects. The width of the $\chi_{c 2}^{\prime}$ was measured to be $(24 \pm 6) \mathrm{MeV}$ [19]. Hence, the branching fraction for the $M 1$ transition $\chi_{c 2}^{\prime} \rightarrow \gamma h_{c}$ is

$$
\mathcal{B}\left(\chi_{c 2}^{\prime} \rightarrow \gamma h_{c}\right)=\mathcal{O}\left(1 \times 10^{-2}\right) .
$$

A future observation with comparable branching fraction would strongly indicate the dominance of coupled-channel effects in the transition. Similar predictions can be made for the other $M 1$ transitions of $P$-wave charmonia. The results for the widths of the transitions $h_{c}^{\prime} \rightarrow \gamma \chi_{c J}$ are shown in Fig. 2(a) as a function of the unknown mass of the $h_{c}^{\prime}$. They should be understood to have an uncertainty of about $40 \%$. One sees that the partial widths of these transitions are of the same order as $\Gamma\left(\chi_{c 2}^{\prime} \rightarrow \gamma h_{c}\right)$, and are typically of $\mathcal{O}(100) \mathrm{keV}$ if using the same model estimates of $g_{1}$ and $g_{1}^{\prime}$ as above, much larger than the results in the potential model [8].

More interestingly, nontrivial parameter-free predictions can be made for the ratios of the partial widths of these hindered $M 1$ transitions to those of the transitions between two $P$-wave charmonia with emission of one pion. In Ref. [1], it is shown that the latter transitions are also dominated by the charmed-meson loops. The one-loop and largest two-loop diagrams scale as $q_{\pi} \Delta / v^{3}$ [1] and $\left(q_{\pi} \Delta / v^{2}\right)\left(M_{D} E_{\pi} / \Lambda_{\chi}^{2}\right)[16]$, respectively, where $q_{\pi}\left(E_{\pi}\right)$ is the pion momentum (energy), and the charged and neutral charmed-meson difference $\Delta$ describes the isospin breaking. One sees that the one-loop diagrams dominate over the two-loop ones. The decay amplitudes and decay widths for the single-pion transitions have been calculated in Ref. [1]. Here we only compare the $M 1$ transitions with the decay
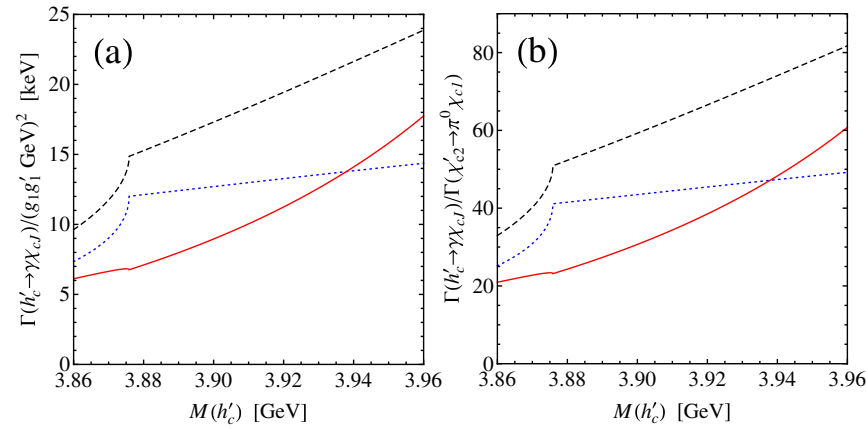

FIG. 2 (color online). (a) Decay widths of the $M 1$ transitions $h_{c}^{\prime} \rightarrow \gamma \chi_{c J}$. (b) Parameter-free ratios $\Gamma\left(h_{c}^{\prime} \rightarrow \gamma \chi_{c J}\right) / \Gamma\left(\chi_{c 2}^{\prime} \rightarrow\right.$ $\left.\pi^{0} \chi_{c 1}\right)$. The dotted, solid, and dashed lines are for $h_{c}^{\prime}$ decays with $\chi_{c 0}, \chi_{c 1}$ and $\chi_{c 2}$ in the final state, respectively. $\chi_{c 2}^{\prime} \rightarrow \chi_{c 1} \pi^{0}$. The width was predicted in Ref. [1] as $(0.29 \pm 0.10)\left(g_{1} g_{1}^{\prime} \mathrm{GeV}\right)^{2} \mathrm{keV}$. The right panel of Fig. 2 shows the parameter-free predictions of the ratios $\Gamma\left(h_{c}^{\prime} \rightarrow\right.$ $\left.\gamma \chi_{c J}\right) / \Gamma\left(\chi_{c 2}^{\prime} \rightarrow \pi^{0} \chi_{c 1}\right)$ as a function of the mass of the $h_{c}^{\prime}$. Such predictions can only be made when both processes are loop-dominated because only in this case the decay amplitudes are proportional to the same product of coupling constants $g_{1} g_{1}^{\prime}$. Were they multipole-dominated, an unknown matrix element of gluon operators would be involved in the transition $\chi_{c 2}^{\prime} \rightarrow \pi^{0} \chi_{c 1}$ so that the process cannot be directly related to the hindered $M 1$ transitions. Even though the uncertainty of these predictions is sizeable, they are markedly different from potential model calculations.

In this Letter, we argue that the hindered $M 1$ transitions of $P$-wave charmonium are dominated by the coupledchannel effects. The conclusion is supported by numerical calculations. With a reasonable estimate of the unknown coupling constants, the results turn out to be much larger than those obtained in the quark model. Parameter-free predictions are made for ratios of partial widths of two completely different types of charmonium transitions: the hindered $M 1$ transitions and single-pion transitions of the $P$-wave charmonia. The $P$-wave charmonia considered here are assumed to be $c \bar{c}$ states so that they are organized as in Eq. (5). If their coupling to the charmed mesons becomes resonant, renormalization is necessary (see, e.g., Refs. $[20,21])$. In that case, the resulting widths for the hindered $M 1$ transitions should still be much larger than the results were the transitions not dominated by the coupled-channel effects, since a resonant coupling tends to enhance the widths further. Experimental efforts on measuring the transitions suggested here are needed towards understanding the coupled-channel effects in the charmonium transitions. In fact, the existing technologies of lattice calculations of the heavy quarkonia radiative transitions [22-24] are well ready to test the picture presented in this Letter: if the hindered $M 1$ transitions of the $P$-wave charmonia are dominated by the coupled-channel effects, the results of simulations with dynamical light quarks should be significantly larger than those in the quenched approximation.

We further notice that the hindered $M 1$ transitions of the $S$-wave charmonia are not well suited for studying the coupled-channel effects. This is because for these transitions the charmed-meson loops are divergent. While the divergence of the triangle diagrams scales as $\mathcal{O}\left(v^{0}\right)$ in the velocity counting, the finite part scales as $\mathcal{O}(v)$. The divergence must be absorbed by a counterterm. However, the counterterm cannot be determined from elsewhere. Hence, parameter-free predictions analogous to those made in this Letter is not possible.

We thank Christoph Hanhart for useful discussions. This work is partially supported by DFG through funds provided to the SFB/TR 16 and the EU I3HP "Study of 
Strongly Interacting Matter" under the Seventh Framework Program of the EU. U.-G. M. also thanks the BMBF for support (Grant No. 06BN9006). F.-K. G. also thanks partial support from the NSFC (Grant No. 11165005). We thank T. Mehen and D.-L. Yang for pointing out an inconsistency in the first version of the manuscript.

*fkguo@hiskp.uni-bonn.de †meissner@hiskp.uni-bonn.de

[1] F.-K. Guo, C. Hanhart, G. Li, U.-G. Meißner, and Q. Zhao, Phys. Rev. D 83, 034013 (2011).

[2] G. Li and Q. Zhao, Phys. Lett. B 670, 55 (2008); Phys. Rev. D 84, 074005 (2011); X. Liu, B. Zhang, and X. Q. Li, Phys. Lett. B 675, 441 (2009); Y. J. Zhang, G. Li, and Q. Zhao, Phys. Rev. Lett. 102, 172001 (2009); for an overview, see Y. J. Zhang, G. Li, and Q. Zhao, Chinese Phys. C 34, 1181 (2010).

[3] E.J. Eichten, talk presented at the 8th International Workshop on Heavy Quarkonium (QWG2011), Darmstadt, Germany, Oct. 04-07, 2011.

[4] E. J. Eichten, K. Lane, and C. Quigg, Phys. Rev. D 73, 014014 (2006).

[5] M. R. Pennington and D. J. Wilson, Phys. Rev. D 76, 077502 (2007); T. Barnes and E. S. Swanson, Phys. Rev. C 77, 055206 (2008); B.-Q. Li, C. Meng, and K.-T. Chao, Phys. Rev. D 80, 014012 (2009).

[6] F.-K. Guo, C. Hanhart, and U.-G. Meißner, Phys. Rev. Lett. 103, 082003 (2009).[104, 109901(E) (2010)].

[7] F.-K. Guo, C. Hanhart, G. Li, U.-G. Meißner, and Q. Zhao, Phys. Rev. D 82, 034025 (2010).

[8] T. Barnes, S. Godfrey, and E. S. Swanson, Phys. Rev. D 72, 054026 (2005).
[9] N. Brambilla, A. Pineda, J. Soto, and A. Vairo, Rev. Mod. Phys. 77, 1423 (2005).

[10] N. Brambilla, Y. Jia, and A. Vairo, Phys. Rev. D 73, 054005 (2006).

[11] V. Bernard, N. Kaiser, J. Gasser, and U.-G. Meißner, Phys. Lett. B 268, 291 (1991).

[12] J.-J. Wu, X.-H. Liu, Q. Zhao, and B.-S. Zou, Phys. Rev. Lett. 108, 081803 (2012).

[13] P. Colangelo, F. De Fazio, and T. N. Pham, Phys. Rev. D 69, 054023 (2004).

[14] J. Hu and T. Mehen, Phys. Rev. D 73, 054003 (2006).

[15] J. F. Amundson, C. G. Boyd, E. E. Jenkins, M.E. Luke, A. V. Manohar, J. L. Rosner, M. J. Savage, and M. B. Wise, Phys. Lett. B 296, 415 (1992).

[16] M. Cleven, F.-K. Guo, C. Hanhart, and U.-G. Meißner, Eur. Phys. J. A 47, 120 (2011).

[17] F.-K. Guo, C. Hanhart, and U.-G. Meißner, Phys. Rev. Lett. 105, 162001 (2010).

[18] G. Burdman and J. F. Donoghue, Phys. Lett. B 280, 287 (1992); M. B. Wise, Phys. Rev. D 45, R2188 (1992); T. M. Yan, H. Y. Cheng, C. Y. Cheung, G. L. Lin, Y. C. Lin, and H.L. Yu, Phys. Rev. D 46, 1148 (1992).55, 5851(E) (1997).

[19] K. Nakamura et al. (Particle Data Group), J. Phys. G 37, 075021 (2010), and 2011 partial update for the 2012 edition.

[20] E. Braaten, M. Kusunoki, and D. Zhang, Ann. Phys. (Leipzig) 323, 1770 (2008).

[21] P. Artoisenet, E. Braaten, and D. Kang, Phys. Rev. D 82, 014013 (2010).

[22] J. J. Dudek, R. G. Edwards, and C. E. Thomas, Phys. Rev. D 79, 094504 (2009).

[23] Y. Chen et al., Phys. Rev. D 84, 034503 (2011).

[24] R. Lewis and R. M. Woloshyn, Phys. Rev. D 84, 094501 (2011). 